\title{
Is Radial Deviation of Wrist Related With Median Nerve Swelling in Patients With Rheumatoid Arthritis? A Radiographic, Ultrasonographic, and Electroneuromyographic Study
}

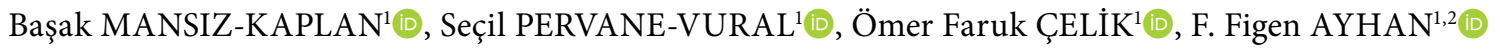 \\ ${ }^{1}$ Department of Physical Medicine and Rehabilitation, University of Health Sciences, Ankara Training and Research Hospital, Ankara, Turkey \\ ${ }^{2}$ Department of Physical Therapy and Rehabilitation, Uşak University, High School of Health Sciences, Uşak, Turkey
}

\begin{abstract}
Objectives: This study aims to investigate whether or not radial deviation developing after wrist involvement of rheumatoid arthritis (RA) is a cause of median nerve swelling.

Patients and methods: The study included 51 RA patients ( 12 males, 39 females; mean age $50.9 \pm 8.9$ years; range, 18 to 65 years) without carpal tunnel syndrome (CTS) detected by electroneuromyography. Duruöz hand index, visual analog scale, and painDETECT questionnaire were performed in clinical assessment. Radiographic measurements including radial inclination (RI) angle were performed. Using ultrasonography, the median nerve cross-sectional areas (CSAs) were measured from the four levels of the distal one third of the forearm, radioulnar joint, pisiform bone, and hook of hamate, while the ulnar nerve CSAs were measured from the pisiform bone.

Results: The study was completed with 102 hands of 51 patients. A negative correlation was found between the RI and the median CSAs measured from the radioulnar joint $(R=-0.49 ; p=0.00)$, the pisiform bone $(R=-0.45 ; p=0.00)$, and hook of hamate $(R=-0.60, p=0.00)$. When the hands were divided into three groups according to the ranges of RI specified in the literature, the median nerve CSA was found to be significantly higher in the group with low $\mathrm{Rl}$ at these levels $(\mathrm{p}<0.001)$.

Conclusion: In patients with RA without CTS, the increase in the median nerve CSAs may be associated with radiographic measures such as radial deviation.

Keywords: Electroneuromyography, median nerve, rheumatoid arthritis, ulnar nerve, ultrasonography, wrist.
\end{abstract}

Rheumatoid arthritis (RA) is a systemic, chronic, and inflammatory disease with the involvement of the synovial joint. Joint involvement is often symmetric, with the most frequently affected being metacarpophalangeal, proximal interphalangeal, and wrist joints. Extra-articular involvement, such as pulmonary fibrosis, subcutaneous nodules, and peripheral neuropathy may be seen in $10-20 \%$ of RA cases. ${ }^{1}$

Carpal tunnel syndrome (CTS) is the most common entrapment neuropathy of the upper extremity and its diagnosis is based on clinical symptoms, physical examination, and nerve conduction studies (NCS). ${ }^{2}$ In recent years, ultrasonography (US) has also been used for the diagnosis of CTS, because US tracking of the median nerve helps detect morphological changes and external compression. ${ }^{3}$ It has been reported that in RA patients without CTS, there is swelling in the median nerve crosssectional areas (CSAs) compared to the healthy population. ${ }^{4}$

Received: December 04, 2018 Accepted: January 13, 2019 Published online: April 22, 2019

Correspondence: Basak Mansiz-Kaplan, MD. SBÜ Ankara Eğitim ve Araştırma Hastanesi, Fiziksel Tıp ve Rehabilitasyon Kliniği, 06230 Altındağ, Ankara, Turkey. Tel: +90 312 - 5953392 e-mail: basakmansiz@hotmail.com

\section{Citation:}

Mansız-Kaplan B, Pervane-Vural S, Çelik ÖF, Ayhan FF. Is radial deviation of wrist related with median nerve swelling in patients with rheumatoid arthritis? A radiographic, ultrasonographic, and electroneuromyographic study. Arch Rheumatol 2019;34(4):380-386. 
The ulnar deviation of the fingers is one of the hand deformities seen in patients with RA, and it has been associated with the radial deviation of the wrist. ${ }^{5}$ We consider that the increase in radial deviation of the wrist may impair balance in the carpal tunnel, thereby increasing the pressure on the median nerve. In this study, we measured the median and ulnar CSAs using US in RA patients without clinical and electrophysiological CTS in order to determine the correlation between the nerve CSAs and the radiographic detection of radial deviation. Our hypothesis was that the increase in radial deviation of the wrist may increase the median nerve CSAs and the decrease in radial deviation of wrist may increase the ulnar CSA. Thus, in this study, we aimed to investigate whether or not radial deviation developing after wrist involvement of RA is a cause of median nerve swelling.

\section{PATIENTS AND METHODS}

A total of 138 patients with a diagnosis of established RA at Ankara Training and Research Hospital Rheumatology Outpatient Clinic between March 2017 and August 2018 were initially evaluated. Exclusion criteria were high disease activity according to disease activity score 28
(DAS28, using erythrocyte sedimentation rate [ESR]), undergoing biological therapy, having a history of trauma to the upper extremity, and the presence of central or peripheral neurological diseases, electroneuromyography (ENMG)-proven CTS, radiculopathy or any other neuropathy, pregnancy or any systemic disease that might cause swelling on nerves (e.g., diabetes mellitus, renal failure, and thyroid disease), US-detected bifid or trifid median nerve, persistent median artery, or space-occupying lesions at the carpal-tunnel. Of the $138 \mathrm{RA}$ patients, 51 (12 males, 39 females; mean age $50.9 \pm 8.9$ years; range, 18 to 65 years) who met the specified criteria and were followed-up at the rheumatology outpatient clinic for at least six months were included in the study (Figure 1). The study protocol was approved by the Ankara Numune Training and Research Hospital Ethics Committee (number of approval: E-17-1619). A written informed consent was obtained from each patient. The study was conducted in accordance with the principles of the Declaration of Helsinki.

Clinical and radiographic evaluations were undertaken by the same physician. Systemic physical, musculoskeletal, and neurological examinations were performed for each patient. The Duruöz hand index (DHI) was applied to

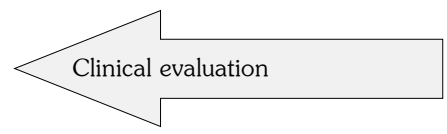

75 patients
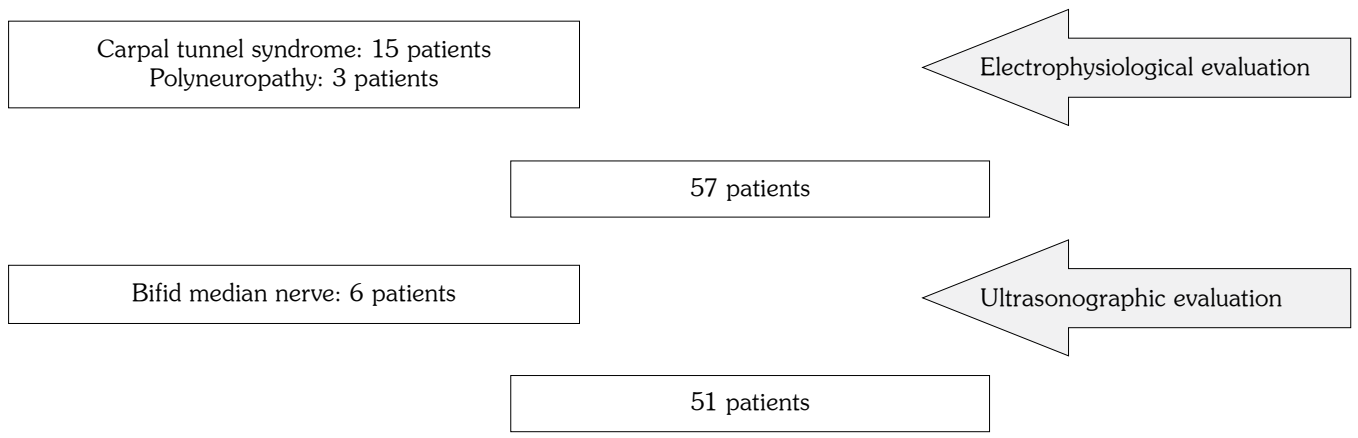

Figure 1. Flowchart of study. 
evaluate the functional disability in both hands. ${ }^{6}$ DHI was first developed to assess functional disability in the hands of RA patients. This scale contains 18 questions concerning the use of hands in daily activities. Each activity is rated by patients from 0 to 5 as follows: 0: "Yes, without difficulty", 1: "Yes, with little difficulty", 2: "Yes, with some difficulty, 3: "Yes, with much difficulty", 4: "Nearly impossible to do", and 5: "Impossible to do".

The presence of pain on the hand was measured by visual analog scale (0-10 points), and whether this pain had neuropathic components was determined using the painDETECT questionnaire. The painDETECT questionnaire consists of four sections to evaluate neuropathic pain. In the first section, the presence of pain is assessed, but not included in scoring. The second section prompts the patient to mark the pain pattern choosing one of the following options: persistent pain with slight fluctuations ( 0 point), persistent pain with pain attacks ( -1 point), pain attacks without pain between them (1 point), pain attacks with pain between them (1 point). In the third section, the pain radiating to other areas is assessed and scored 2 points if present. The last section contains seven items on a six-point scale: never 0 , hardly noticed 1 , slightly 2 , moderately 3 , strongly 4 , and very strongly 5 . A final score of 12 or below represents no neuropathic pain, 12-19 suggests that the result is ambiguous, and 20 or above indicates presence of neuropathic components. ${ }^{7}$ The Turkish validity and reliability analyses of the questionnaire were undertaken by Alkan et al. ${ }^{8}$

Anteroposterior radiography was performed on both hands of all patients by the same technician using the standard technique. Radial inclination (RI) is the angle between the distal surface and the longitudinal axis of the radius. The normal range of $\mathrm{RI}$ specified in the literature is $21^{\circ}$ to $25^{\circ} .9^{9}$ Radial angulation of the wrist (RAW) is the angle between the radial corner of the second metacarpal bone and the distal radius surface. ${ }^{10}$ The angle of phalangeal drift (APD) is measured at the intersection of the lines along the shafts of the second metacarpal bone and the second proximal phalanx. As APD increases, the ulnar deviation of the fingers increases. ${ }^{11}$ The radiographic measurements were repeated three times for each patient and averaged. The measurements of RI, RAW, and APD were shown in Figure 2.

Nerve US was performed by the same clinician with five years of experience in this area. All the US evaluations were performed with a 7-12 $\mathrm{MHz}$ (General Electric, LOGIQ P5, Oklahoma City, USA) linear array probe with the patient in a supine position. The nerves were viewed in the axial plane. The transducer was kept perpendicular to the nerve. The median nerve CSAs were measured at the distal one third of the forearm, hook of hamate, pisiform bone, and radio-ulnar joint. The ulnar nerve CSA was measured at the level of the pisiform bone. ${ }^{3}$ The CSA measurements were undertaken by tracing the nerve just inside its hyperechoic rim (Figure 3). Three different measurements were obtained and the average of the three was used for each level.

Nerve conduction studies for median and ulnar nerves were performed by the same clinician with four years of experience in ENMG using the Nihon Kohden Neuropack M1 system
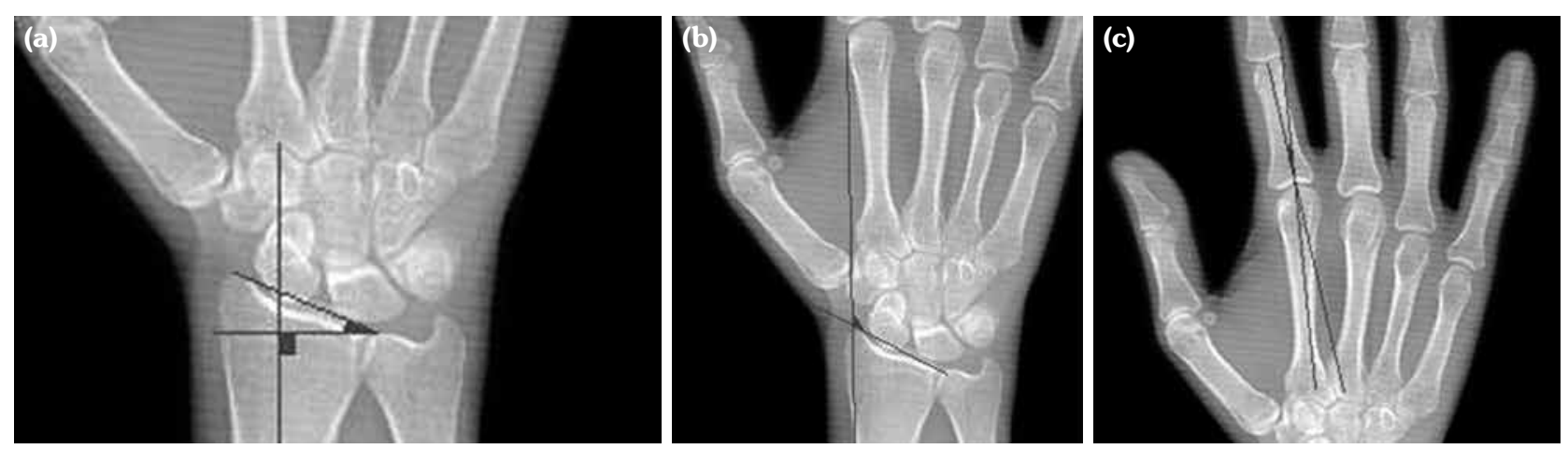

Figure 2. (a) Radial inclination. (b) Radial angulation of wrist. (c) Angle of phalangeal drift. 

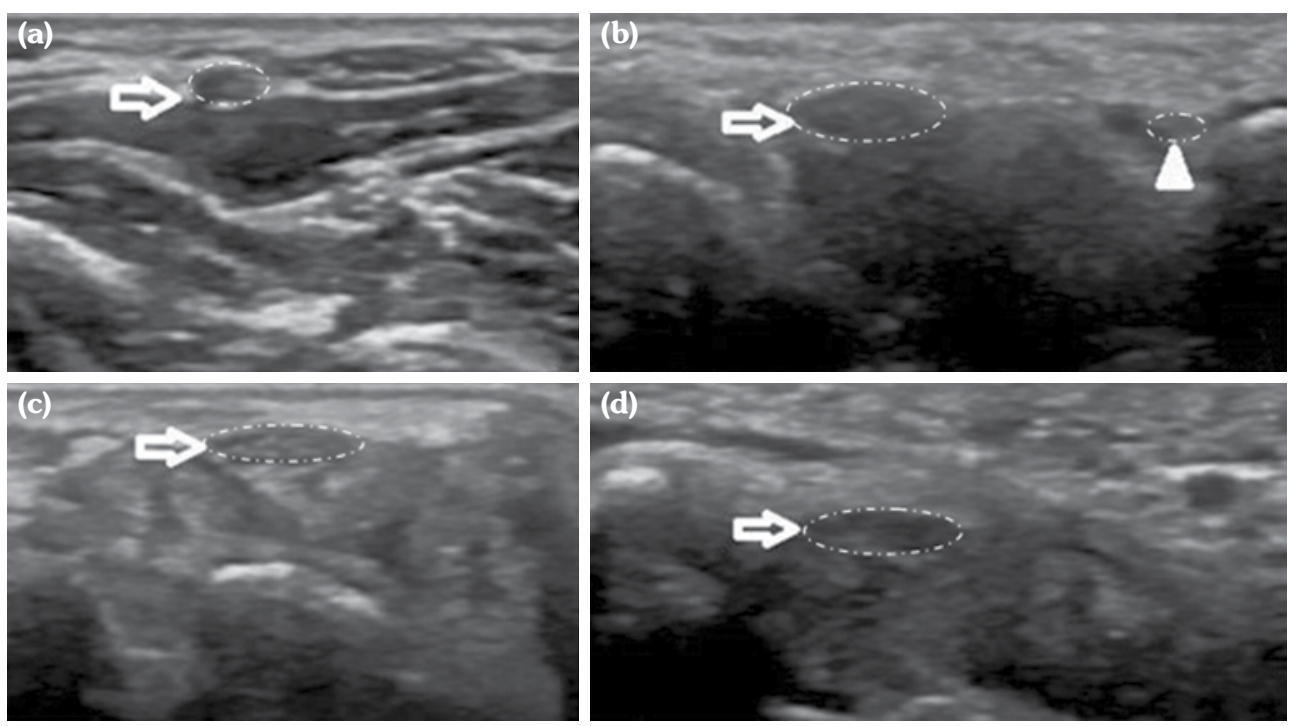

Figure 3. Transverse sonogram of median and ulnar nerve cross sectional area with ultrasonography. (a) Level of distal one third forearm. (b) Level of radio-ulnar joint. (c) Level of pisiform bone. (d) Level of hook of hamate. Arrow: Median.

(Nihon Inc., Tokyo, Japan). The room temperature was maintained at $25^{\circ}$ and hand temperature at $32^{\circ}$ and above. The median motor and sensory nerve conduction studies, and the ulnar nerve conduction studies were performed, and the median and ulnar nerve latency differences were measured from the second dorsal interosseous muscles. In cases where necessary, the ulnar, peroneal, and tibial nerve motor conduction studies, the sural nerve sensory studies, and needle ENMG were conducted to exclude radiculopathy or polyneuropathy. The presence of CTS was assessed according to the American Association of Neuromuscular \& Electrodiagnostic Medicine guidelines. ${ }^{12}$

\section{Statistical analysis}

The statistical analyses were performed with the SPSS version 15.0 program (SPSS Inc., Chicago, IL, USA). Descriptive statistics were obtained as mean \pm standard deviation for continuous variables and as numbers ( $\mathrm{n}$ ) for categorical

Table 1. Demographic and clinical features of patients

\begin{tabular}{|c|c|c|c|c|}
\hline & $\mathrm{n}$ & $\%$ & Mean \pm SD & Min-Max \\
\hline Age (year) & & & $50.9 \pm 8.9$ & $27.0-65.0$ \\
\hline Body mass index $\left(\mathrm{kg} / \mathrm{m}^{2}\right)$ & & & $28.1 \pm 4.1$ & $19.4-36.8$ \\
\hline Duration of RA (year) & & & $13.0 \pm 8.7$ & $1.0-37.0$ \\
\hline DAS28 score & & & $2.4 \pm 0.8$ & $0.6-4.6$ \\
\hline Duruöz hand index & & & $12.3 \pm 18.6$ & $0-72$ \\
\hline PainDETECT questionnaire & & & $0.5 \pm 1.0$ & $0-5$ \\
\hline VAS-pain (cm) & & & $1.8 \pm 2.4$ & $0-8$ \\
\hline \multicolumn{5}{|l|}{ Sex } \\
\hline Females & 39 & 76.5 & & \\
\hline Hand dominance (right) & 47 & 92.2 & & \\
\hline Low level of education & 32 & 62.7 & & \\
\hline Rheumatoid factor positivity & 33 & 64.7 & & \\
\hline
\end{tabular}


Table 2. Nerve cross-sectional areas of all patients

\begin{tabular}{lcc}
\hline & Mean \pm SD & Min-Max \\
\hline Forearm-median nerve CSA $\left(\mathrm{mm}^{2}\right)$ & $6.2 \pm 1.4$ & $4-13$ \\
Distal RUJ-median nerve CSA $\left(\mathrm{mm}^{2}\right)$ & $8.2 \pm 1.6$ & $5-14$ \\
Hamate-median nerve CSA $\left(\mathrm{mm}^{2}\right)$ & $8.4 \pm 1.6$ & $5-16$ \\
Hamatum-median nerve CSA $\left(\mathrm{mm}^{2}\right)$ & $8.7 \pm 1.8$ & $5-16$ \\
Hamate-ulnar nerve CSA $\left(\mathrm{mm}^{2}\right)$ & $4.1 \pm 0.9$ & $3-10$ \\
\hline
\end{tabular}

SD: Standard deviation; Min: Minimum; Max: Maximum; CSA: Crosssectional area; RUJ: Radioulnar joint.

variables. The Shapiro-Wilk test was performed to check the normal distribution. A one-way analysis of variance test was applied to the quantitative data for the comparison of three or more groups, which showed a normal distribution, and the group that showed a difference was detected by the Tukey honestly significant difference and Tamhane tests. The Kruskal Wallis test was also used in the comparison of three or more groups without a normal distribution. To evaluate the correlation between the variables, the Pearson and Spearman correlation analyses were used for data with or without a normal distribution, respectively. A Pearson Chi-square test was used to test the differences in proportions. The statistical significance was evaluated at the $p<0.01$ and $\mathrm{p}<0.05$ levels.

\section{RESULTS}

The mean age of the patients was $50.9 \pm 8.9$ years and the mean duration of symptoms was $13.0 \pm 8.7$ years. The demographic data and general characteristics of the patients were summarized in Table 1 . According to the US assessment of 102 hands, 27 hands met the CTS criterion of a median nerve CSA of $\geq 10 \mathrm{~mm}^{2}$ specified in the literature. ${ }^{13}$ Thus, $27 \%$ of the RA patients with no clinical or electrophysiological findings were diagnosed with CTS according to US. The US measurements of median and ulnar nerve CSAs of the patients were shown in Table 2.

Analyzing radiographic measurements with US, electrophysiological and clinical assessments, a negative correlation was found between RI and the median CSAs measured at the three levels of the radioulnar joint (RUJ) $(\mathrm{R}=-0.49$, $\mathrm{p}=0.00)$, pisiform bone $(\mathrm{R}=-0.45, \mathrm{p}=0.00)$, and hook of hamate $(\mathrm{R}=-0.60 ; \mathrm{p}=0.00)$. However, no correlation was found between the median nerve CSAs and C-reactive protein (CRP), ESR, DAS28, age, weight, height, painDETECT scores, DHI, or duration of RA symptoms. There were also positive correlations between APD and duration of RA ( $\mathrm{R}=0.39 ; \mathrm{p}=0.00)$; $\mathrm{APD}$ and median motor latency $(R=0.37 ; p=0.00)$.

The 102 hands were divided into three groups according to the RI ranges provided in the literature: group 1 with 45 normal hands ( $\mathrm{RI}=21-25)$, group 2 with 38 radially deviated hands $(\mathrm{RI}<21)$, and group 3 with 19 ulnar deviated hands $(\mathrm{RI}>25)$. In these three groups, there was no significant difference between the results of NCS and occupation, educational status, dominant hand use, age, ESR, CRP, DAS28, painDETECT scores, DHI or duration of RA symptoms ( $p>0.05)$. When the groups

Table 3. Comparison of ultrasonography-nerve cross-sectional area in three groups according to radial inclination

\begin{tabular}{|c|c|c|c|c|c|c|c|c|}
\hline \multirow[b]{2}{*}{ Cross-sectional area $\left(\mathrm{mm}^{2}\right)$} & \multicolumn{2}{|c|}{ Group $1(n=45)$} & \multicolumn{2}{|c|}{ Group $2(n=38)$} & \multicolumn{2}{|c|}{ Group $3(n=19)$} & \multirow[b]{2}{*}{$p$} & \multirow[b]{2}{*}{$\begin{array}{c}\text { Post-hoc } \\
\text { comparisons }\end{array}$} \\
\hline & Mean \pm SD & Min-Max & Mean \pm SD & Min-Max & Mean \pm SD & Min-Max & & \\
\hline Forearm-median nerve & $6.4 \pm 1.6$ & $4-13$ & $6.3 \pm 1.1$ & $5-9$ & $5.8 \pm 1.6$ & $4-9$ & 0.41 & \\
\hline Distal RUJ-median nerve & $7.9 \pm 1.6$ & $5-14$ & $9.1 \pm 1.4$ & $6-13$ & $7.1 \pm 1.1$ & $5-9$ & $0.00^{*}$ & $\begin{array}{l}2>1 \\
2>3\end{array}$ \\
\hline Pisiform-median nerve & $8.0 \pm 1.7$ & $5-16$ & $9.3 \pm 1.1$ & $6-12$ & $7.6 \pm 1.7$ & $6-13$ & $0.00^{*}$ & $\begin{array}{l}2>1 \\
2>3\end{array}$ \\
\hline Hook of hamate-median nerve & $8.2 \pm 1.6$ & $5-16$ & $9.9 \pm 1.4$ & $7-15$ & $7.5 \pm 1.5$ & $6-13$ & $0.00^{*}$ & $\begin{array}{l}2>1 \\
2>3\end{array}$ \\
\hline Pisiform-ulnar nerve & $4.2 \pm 1.2$ & $3-10$ & $4.1 \pm 0.7$ & $3-5$ & $4.0 \pm 0.8$ & $3-6$ & 0.72 & \\
\hline
\end{tabular}


were compared according to disease-modifying anti-rheumatic drug therapy, leflunomide use was significantly higher in group $3(p=0.001)$. The median CSA measurements of the groups significantly differed at the levels of the RUJ, pisiform bone, and hook of hamate $(p<0.001)$ (Table 3).

\section{DISCUSSION}

We found that as radial deviation increased, the median nerve CSAs also increased at the levels of the RUJ, pisiform bone, and hook of hamate in this study, which, to our knowledge, was not reported before. CTS was determined according to nerve conduction studies in 15 of 138 patients, and these patients were excluded. After that, CTS was diagnosed using US in 27 of 102 hands included in the study. Interestingly, none of the patients had neuropathic pain profile using painDETECT.

Hammer et al. ${ }^{14}$ compared RA patients clinically suspected of CTS with healthy controls and demonstrated increased median CSA on the US of the median nerve. In a later study, the same authors compared RA patients having no clinical symptoms or findings of CTS with a control group, but this time they observed no increase in the median nerve CSA. ${ }^{15}$ In a recent study, 30 RA patients without clinical or electrophysiological CTS signs and 30 healthy individuals were evaluated in terms of not only the median nerve CSA but also the ulnar nerve CSA measured from various levels to the elbow. The authors reported a significant increase in the median nerve CSAs at the levels of the RUJ, pisiform bone, and hook of hamate compared to the control group. ${ }^{4}$ Although we were not able to make such a comparison due to not having a control group, we observed that the measurements taken at the same levels were correlated with RI. That is, as the deviation of the wrist increased toward the radial, the swelling in the median nerve also increased. These levels fit the proximal entrance and exit of the carpal tunnel, which is located between the carpal bones and the flexor retinaculum, through which the flexor muscle tendons and the median nerve pass. Any change in one of these structures adjacent to the median nerve can change the pressure in the tunnel. It is known that as RI decreases (i.e., radial deviation increases), the load on the carpal bones, particularly the lunate bone, increases. ${ }^{9}$ This increased load may cause elevated pressure in the carpal tunnel. However, pressure increase in the carpal tunnel has also been reported when the wrist is at the position of radial deviation (albeit not as much as flexion and extension). ${ }^{16}$ Since muscle movements may also have an effect on this increase in pressure, it is difficult to identify bone deformity as being solely responsible for radial deviation; however, the contribution of this deviation to increased pressure cannot be denied. Depending on the rise of pressure in the carpal tunnel, we may have detected an increase in the median nerve CSAs because if the nerve is entrapped for any reason (increased pressure, local pressure, etc.), nerve swelling may occur even before the entrapment of the nerve. ${ }^{17}$

In addition to the median nerve, US evidence of swelling in the ulnar nerve has also been reported in RA patients compared to the healthy population. ${ }^{4}$ According to our hypothesis, as the ulnar deviation increases, the ulnar nerve CSA would increase; however, we did not find any such correlation in this study. We may attribute this to the small number of patients having RI of $>25^{\circ}$.

In the literature, studies about nerve conduction, though not together with US, mostly reported no correlation between clinical symptoms and the severity of nerve involvement. Age, weight, height, ESR, CRP, disease activity, or disease duration were not associated with median nerve involvement in an investigation of CTS in RA. ${ }^{18}$ In our study, we also compared clinical and laboratory data with the median nerve CSAs, and we found no correlation between the median nerve CSA and age, weight, height, disease activity, ESR, CRP, duration of disease, hand functional disability, or NCS similar to the literature.

The strengths of our study were that our inclusion criteria were stringent and we used not only NCS but also US to detect CTS. The main limitation of our study was that we did not have any control group. The relationship between RI and the median nerve CSA in a healthy population is a subject that needs to be investigated in future studies.

In conclusion, to our knowledge, this is the first study that evaluated the relationship between radiographic measures and nerve CSAs in 
patients with RA. According to our findings, as $\mathrm{RI}$ decreases or radial deviation increases, the median nerve CSAs increase in patients with RA.

\section{Declaration of conflicting interests}

The authors declared no conflicts of interest with respect to the authorship and/or publication of this article.

\section{Funding}

The authors received no financial support for the research and/or authorship of this article.

\section{REFERENCES}

1. Turesson C, O'Fallon WM, Crowson CS, Gabriel SE, Matteson EL. Occurrence of extraarticular disease manifestations is associated with excess mortality in a community based cohort of patients with rheumatoid arthritis. J Rheumatol 2002;29:62-7.

2. Bland JD. Carpal tunnel syndrome. Curr Opin Neurol 2005;18:361-4.

3. Wu CH, Chang KV, Özçakar L, Hsiao MY, Hung CY, Shyu SG, et al. Sonographic tracking of the upper limb peripheral nerves: a pictorial essay and video demonstration. Am J Phys Med Rehabil 2015;94:740-7.

4. Yagci I, Akdeniz Leblebicier M, Mansiz Kaplan B, Ozturk Gokbakan D, Akyuz G. Sonographic Measurements Can Be Misleading for Diagnosing Carpal Tunnel Syndrome in Patients with Rheumatoid Arthritis. Acta Reumatol Port 2016;41:40-4.

5. Shapiro JS. A new factor in the etiology of ulnar drift. Clin Orthop Relat Res 1970;68:32-43.

6. Duruöz MT, Poiraudeau S, Fermanian J, Menkes CJ, Amor B, Dougados M, et al. Development and validation of a rheumatoid hand functional disability scale that assesses functional handicap. J Rheumatol 1996;23:1167-72.

7. Freynhagen R, Baron R, Gockel U, Tölle TR. painDETECT: a new screening questionnaire to identify neuropathic components in patients with back pain. Curr Med Res Opin 2006;22:1911-20.

8. Alkan H, Ardic F, Erdogan C, Sahin F, Sarsan A, Findikoglu G. Turkish version of the painDETECT questionnaire in the assessment of neuropathic pain: a validity and reliability study. Pain Med 2013;14:1933-43.

9. Broder J. Diagnostic imaging for the emergency physician. Philadelphia: Elsevier Healh Science; 2011.

10. Ito J, Koshino T, Okamoto R, Saito T. Radiologic evaluation of the rheumatoid hand after synovectomy and extensor carpi radialis longus transfer to extensor carpi ulnaris. J Hand Surg Am 2003;28:585-90.

11. Read GO, Solomon L, Biddulph S. Relationship between finger and wrist deformities in rheumatoid arthritis. Ann Rheum Dis 1983;42:619-25.

12. Werner RA, Andary M. Electrodiagnostic evaluation of carpal tunnel syndrome. Muscle Nerve 2011;44:597-607.

13. El Miedany YM, Aty SA, Ashour S. Ultrasonography versus nerve conduction study in patients with carpal tunnel syndrome: substantive or complementary tests. Rheumatology (Oxford) 2004;43:887-95.

14. Hammer HB, Hovden IA, Haavardsholm EA, Kvien TK. Ultrasonography shows increased cross-sectional area of the median nerve in patients with arthritis and carpal tunnel syndrome. Rheumatology (Oxford) 2006;45:584-8.

15. Hammer HB, Haavardsholm EA, Kvien TK. Ultrasonographic measurement of the median nerve in patients with rheumatoid arthritis without symptoms or signs of carpal tunnel syndrome. Ann Rheum Dis 2007;66:825-7.

16. Werner R, Armstrong TJ, Bir C, Aylard MK. Intracarpal canal pressures: the role of finger, hand, wrist and forearm position. Clin Biomech (Bristol, Avon) 1997:12:44-51.

17. Lawande $\mathrm{AD}$, Warrier SS, Joshi MS. Role of ultrasound in evaluation of peripheral nerves. Indian $\mathrm{J}$ Radiol Imaging 2014;24:254-8.

18. Sakthiswary R, Singh R. Has the median nerve involvement in rheumatoid arthritis been overemphasized? Rev Bras Reumatol Engl Ed 2017;57:122-8. 